\title{
Instability of vortical and acoustic modes in supersonic round jets
}

\author{
K. H. Luo and N. D. Sandham \\ Department of Engineering, Queen Mary and Westfield College, London E1 4NS, United Kingdom
}

(Received 15 August 1995; accepted 4 November 1996)

\begin{abstract}
The stability of "top-hat" and fully developed jet profiles is investigated by an inviscid linear stability theory for compressible flow. The study covers a wide range of the Mach number and the temperature ratio. Two types of instabilities are found: vortical and acoustic, each of which can be subdivided into non-radiating (subsonic) and radiating (supersonic) modes. The vortical mode is the continuation of the Kelvin-Helmholtz instability from incompressible flow. The acoustic mode is a compressible flow phenomenon, which becomes important at large Mach numbers. Temperature-ratio effects can be destabilizing or stabilizing, depending on the Mach number and mode of instability. A spectrum of unstable acoustic modes, including axisymmetric ones, are found to exist in the fully developed jet. For this jet, acoustic axisymmetric waves become more unstable than both vortical and acoustic helical waves at Mach numbers over about 3. Strong evidence of a resonance mechanism for acoustic modes is seen in the growth rate curves at high Mach numbers, where a spectrum of local peaks and valleys appears at regularly distributed frequencies. (c) 1997 American Institute of Physics. [S1070-6631(97)01604-8]
\end{abstract}

\section{INTRODUCTION}

The linear stability of a laminar jet subject to small disturbances has been studied for many years and is still of theoretical and practical interest. There has been ample evidence that instability waves are major sources of noise in supersonic jets. ${ }^{1-6}$ The noise generation is most efficient when the phase speed of an instability wave is supersonic relative to the ambient fluid and intense Mach waves are emitted. $^{7-9}$ Therefore, determination of stability characteristics of supersonic jets is a vital step for the prediction of jet noise, where compressibility often plays an important role.

The flow field of a jet issuing from a circular orifice into an ambient fluid can be roughly divided into a potential core region, a transition region and a fully developed region. At the beginning of the core region, the shear layer is very thin and often modeled by a vortex sheet, as in the theoretical studies of Gill, ${ }^{10}$ Zaninetti ${ }^{11,12}$ and Tam and Hu. ${ }^{4}$ The "tophat" profile also belongs to the potential core region with a thin but finite shear layer. This region can be represented by hyperbolic-tangent functions. ${ }^{13}$ The fully developed (selfsimilar) region can be represented by a Gaussian or a parabolic profile. The studies of Batchelor and Gill, ${ }^{14}$ Lessen and Singh $^{15}$ and Morris ${ }^{16}$ all chose the same parabolic profile. The profile is characterized by a thick shear layer with slowly varying velocity.

Previous studies on jet stability have been focused on the potential core region, partly because the jet is most unstable there and partly because the problem is amenable to theoretical analysis using the vortex sheet assumption. Two types of instability modes have been found: vortical and acoustic. The vortical mode, also called "vorticity mode" by Mack, ${ }^{17}$ is due to the Kelvin-Helmholtz instability, which is the primary hydrodynamic instability. The term "vortical mode" is used to indicate the extension of Kelvin-Helmholtz instability into the compressible regime. A comprehensive review of findings on vortical mode instability under various jet flow conditions has been given by Michalke. ${ }^{13}$ The acoustic mode instability was first discovered by $\mathrm{Mack}^{18}$ in compressible boundary layers and by Gill ${ }^{10}$ in jets and wakes. The term "acoustic mode" originally referred to neutral sound waves reflecting back and forth between the wall and the sonic line where the relative Mach number is equal to unity. Mack ${ }^{17}$ extended the terminology to include stable, neutral and amplified waves, which arose when there was an embedded region of local supersonic flow relative to the phase speed of the instability wave. It is noted that $\mathrm{Tam}$ and $\mathrm{Hu}^{4}$ used subsonic and supersonic modes to designate the acoustic modes with subsonic and supersonic relative Mach numbers respectively. The properties of acoustic mode were extensively discussed by Mack ${ }^{17}$ based on linear stability analysis of twodimensional waves. Tam and $\mathrm{Hu}^{4}$ offered a physical explanation of the origin of acoustic mode based on the pressure imbalance across a vortex sheet. For a round jet with finite thickness, no rigorous theory exists to provide a clear physical interpretation of amplified acoustic modes.

Jet stability in the fully developed region has received relatively little attention in the past. Batchelor and Gill ${ }^{14}$ proved mathematically that unstable axisymmetric waves were excluded from the fully developed incompressible jet with the parabolic profile, since it has no inflection point. Numerical calculations of Lessen and Singh ${ }^{15}$ and Morris ${ }^{16}$ confirmed the above statement and further established that the first helical mode was the most unstable. Since compressibility was not included, these studies ruled out the possibility of the existence of unstable acoustic waves. This possibility is fully explored in the present study, which will show that unstable axisymmetric waves not only exist for the parabolic profile at high Mach numbers but also can become more unstable than helical waves.

In the next section, the stability problem is defined and numerical methods for solving the problem are outlined. In Sec. III, the necessary conditions for instability are revisited. This is followed by presentation of results from a "top-hat" profile in Sec. IV. The temperature-ratio effects on stability are treated in Sec. V. The stability results from a fully de- 
veloped profile are given in Sec. VI. Finally, discussions and conclusions are presented in Secs. VII and VIII.

\section{PRELIMINARIES}

We consider the inviscid linear stability problem governed by the compressible Euler equations in the cylindrical coordinate system $(r, \theta, z)$. A set of coupled first-order linearized equations for the six dependent variables $\Phi=u_{r}, u_{\theta}, u_{z}, \rho, p, T$ can be derived using the normal-mode, parallel-flow approach. Solutions of disturbances are sought in the following form:

$$
\Phi^{\prime}(r, \theta, z, t)=\hat{\Phi}(r) e^{i(\alpha z+m \theta-\omega t)},
$$

where $\alpha, m$ and $\omega$ are the axial wavenumber, azimuthal wavenumber and frequency, respectively. With some mathematical manipulation, the six linearized equations can be reduced to a single disturbance equation for pressure:

$$
D^{2} \hat{p}+A D \hat{p}+B \hat{p}=0,
$$

where $D \equiv d / d r$ and

$$
\begin{aligned}
& A=\frac{1}{r}-D \ln \bar{\rho}-\frac{2 \alpha D \bar{U}_{z}}{\alpha \bar{U}_{z}-\omega}+\frac{1}{\operatorname{Fr}} \frac{i \alpha D \ln \bar{\rho}}{\left(\alpha \bar{U}_{z}-\omega\right)^{2}}, \\
& B=\bar{\rho} M_{1}^{2}\left(\alpha \bar{U}_{z}-\omega\right)^{2}-\alpha^{2}-\frac{m^{2}}{r^{2}}-\frac{i \alpha \bar{\rho}_{1}^{2}}{\mathrm{Fr}} .
\end{aligned}
$$

The boundary conditions are

$$
\begin{aligned}
& \hat{p}(r \rightarrow 0)=C_{1} I_{m}(\beta r), \\
& \hat{p}(r \rightarrow \infty)=C_{2} K_{m}(\beta r),
\end{aligned}
$$

where $I_{m}$ and $K_{m}$ are the modified Bessel functions of the first and second kind of order $m$ and

$$
\beta^{2}=\alpha^{2}-\bar{\rho}_{1}^{2}\left(\alpha \bar{U}_{z}-\omega\right)^{2}+\frac{i \alpha \bar{\rho} M_{1}^{2}}{F r} .
$$

In the above equations and all the following discussions, all variables are normalized with their jet centerline values. The length is normalized with the jet radius where the axial velocity is equal to the mean of the two free-streams $R_{1 / 2}^{*}$ where the superscript $*$ indicates a dimensional quantity. A bar over a variable denotes its mean value. $M_{1}$ is the jet Mach number. $\mathrm{Fr}$ is the Froude number, which is a nondimensional gravitational force. When it is neglected $(F r \rightarrow \infty)$, as is the case in this study, Eq. (2) reduces to the form obtained by Michalke. ${ }^{13}$

In the temporal stability analysis, $\alpha$ is real and $\omega$ complex. In the spatial analysis, $\alpha$ is complex and $\omega$ real. The resulting eigenvalue problems for the pressure disturbance are solved by a two-domain shooting method, with integration by a variable-step fifth order Runge-Kutta scheme.

Two jet profiles were investigated:

$$
\begin{aligned}
& \bar{U}_{z}=0.5\left\{1+\tanh \left[0.5 R_{z}(1-r)\right]\right\}, \\
& \bar{U}_{z}=\left[1+(\sqrt{2}-1) r^{2}\right]^{-2},
\end{aligned}
$$

where the jet parameter $R_{z}=R_{1 / 2}^{*} / \theta^{*}=1 / \theta$ characterizes jet profiles at different axial positions. $\theta^{*}$ is the dimensional momentum thickness. The first is called a "top-hat" profile, which represents the initial mean velocity near the jet exit. It has been investigated by Michalke ${ }^{13}$ and Morris, ${ }^{16}$ among others. The second profile is a similarity solution of a steady jet emerging from a small circular orifice into an unbounded fluid. ${ }^{19}$ Experiments have also confirmed that the mean velocity profile approaches the form in Eq. (7) far from the jet exit. It thus has been widely used to represent the fully developed jet downstream of the potential core. ${ }^{13-16}$ The mean temperature was calculated with a Crocco-Busemann relation for unity Prandtl number:

$$
\begin{aligned}
\bar{T}= & M_{1}^{2} \frac{(\gamma-1)}{2}\left(u\left(1+\bar{U}_{2}\right)-u^{2}-\bar{U}_{2}\right)+\frac{\bar{T}_{2}(1-u)}{\left(1-\bar{U}_{2}\right)} \\
& +\frac{\left(u-\bar{U}_{2}\right)}{\left(1-\bar{U}_{2}\right)}
\end{aligned}
$$

where $\gamma$ is the ratio of specific heats $c_{p} / c_{v}$ and subscripts 1 and 2 indicate the jet centerline and the ambient fluids, respectively. It is noted that the temperature profile depends on the Mach number in compressible flow. The above profiles are considered in this study to give typical stability characteristics. Quantitative information about growth rates at very high Mach numbers could be obtained with experimentally determined profiles of velocity and temperature.

\section{REVISIT OF NECESSARY CONDITIONS FOR INSTABILITY}

A necessary condition for instability of a velocity profile was first given by Rayleigh in $1892 .{ }^{20}$ His theorem states that the presence of at least one inflection point $\left(\bar{U}^{\prime \prime}=0\right)$ in the velocity profile is necessary for instability. Here, the prime indicates differentiation with respect to the relevant coordinate. Fjørtoft ${ }^{21}$ later showed that furthermore $\bar{U}^{\prime \prime}$ must change sign around the inflection point. Batchelor and Gill ${ }^{14}$ derived the above conditions in a cylindrical coordinate system. However, these conditions have been reached for inviscid, incompressible, isothermal flow. Lees and $\mathrm{Lin}^{22}$ considered the stability conditions for inviscid compressible boundary layers. The general stability criteria obtained were stated by Lees ${ }^{23}$ as follows: “(1) If the quantity $\left(\bar{\rho} \bar{U}^{\prime}\right)^{\prime}$ vanishes for some value of $\bar{U}>1-1 / M_{1}$, then neutral and selfexcited subsonic disturbances exist and the inviscid compressible flow is unstable. (2) If the quantity $\left(\bar{\rho} \bar{U}^{\prime}\right)^{\prime}$ does not vanish for some value of $\bar{U}>1-1 / M_{1}$, then all subsonic disturbances of finite wavelength are damped and the inviscid compressible flow is stable.' Criteria (1) and (2) thus give the sufficient and necessary conditions of instability, i.e., $\left(\bar{\rho} \bar{U}^{\prime}\right)^{\prime}=0$ at some interior point. It is noted, however, that Lees and $\mathrm{Lin}^{22}$ only proved rigorously the sufficient condition of instability (1). The point where $\left(\bar{\rho} \bar{U}^{\prime}\right)^{\prime}=0$ is often called a "generalized inflection point." Whether or not the necessary condition (2) is valid for compressible flow is still an open question. Numerical findings in Sec. VI of amplified subsonic waves in the absence of a generalized inflection 
point are apparently in contradiction of (2), which suggests that further analysis of instability conditions for compressible flow is needed.

The disturbance equation for the radial velocity component is written in the cylindrical coordinate system as:

$$
\begin{aligned}
& D\left[\frac{\bar{\rho} r}{m^{2}+\alpha^{2} r^{2}} G\left(r, M_{1}\right) D \Lambda\right] \\
& -\frac{\alpha \Lambda}{\alpha \bar{U}_{z}-\omega} D\left[\frac{r \bar{\rho} D \bar{U}_{z}}{m^{2}+\alpha^{2} r^{2}} G\left(r, M_{1}\right)\right]-\frac{\bar{\rho}}{r} \Lambda=0,
\end{aligned}
$$

where $\Lambda=r \hat{u}_{r}$ and

$$
\begin{aligned}
& G\left(r, M_{1}\right)=\frac{m^{2}+\alpha^{2} r^{2}}{F\left(r, M_{1}\right)}, \\
& F\left(r, M_{1}\right)=m^{2}+\alpha^{2} r^{2}-\bar{\rho}\left(\alpha \bar{U}_{z}-\omega\right)^{2} r^{2} M_{1}^{2} .
\end{aligned}
$$

Assuming a temporal stability problem, necessary conditions for instability can be obtained by the following wellestablished procedures: ${ }^{14}$ (a) multiplication of Eq. (9) by the complex conjugate of $\Lambda$ and then (b) integration by parts over the domain $(0, \infty)$. By noting $\Lambda=0$ at $r=0$ and $\infty$, the imaginary part of the resulting equation gives

$$
\omega_{i}\left(I_{1}+I_{2}-I_{3}\right)=0,
$$

where

$$
\begin{aligned}
I_{1}= & \int_{0}^{\infty} 2 M_{1}^{2} \Pi \frac{r^{3} \bar{\rho}^{2}}{\left|F\left(r, M_{1}\right)\right|^{2}}\left[|D \Lambda|^{2}+\frac{\alpha^{2}\left(D \bar{U}_{z}\right)^{2}|\Lambda|^{2}}{\left|\alpha \bar{U}_{z}-\omega\right|^{2}}\right] d r, \\
I_{2}= & \int_{0}^{\infty} 2 M_{1}^{2} \Pi^{2} \frac{\alpha|\Lambda|^{2}}{\left|\alpha \bar{U}_{z}-\omega\right|^{2}} D\left[\frac{r^{3} \bar{\rho}^{2} D \bar{U}_{z}}{\left|F\left(r, M_{1}\right)\right|^{2}}\right] d r, \\
I_{3}= & \int_{0}^{\infty} \frac{\alpha|\Lambda|^{2}}{\left|\alpha \bar{U}_{z}-\omega\right|^{2}} \\
& \times D\left[\left\{\Upsilon-\left(\Pi^{2}-\omega_{i}^{2}\right) \bar{\rho} r^{2} M_{1}^{2}\right\} \frac{r \bar{\rho} D \bar{U}_{z}}{\left|F\left(r, M_{1}\right)\right|^{2}}\right] d r,
\end{aligned}
$$

where $\Pi=\alpha \bar{U}_{z}-\omega_{r}, Y=m^{2}+\alpha^{2} r^{2}$ and $|\cdot|$ denotes the magnitude of a complex variable. In order for instability to exist ( $\left.\omega_{i} \neq 0\right)$, a necessary condition is $I_{3} \equiv I_{1}+I_{2}$. As can be seen, satisfaction of this condition depends not only on the mean velocity and density profiles but also the jet Mach number and the disturbance wavenumber. This fact highlights the difficulty of reaching a general instability condition for fully compressible flow. However, if $M_{1} \rightarrow 0$, then $I_{1}=I_{2}=0$ and $I_{3}$ must be zero for instability. This leads to the first necessary condition for instability: (A) There must be an interior point at which $D \Omega(r)=0$, where $\Omega(r)$ $=r \bar{\rho} D \bar{U}_{z} /\left(m^{2}+\alpha^{2} r^{2}\right)$. This is the counterpart in the cylindrical coordinate system of condition (2) of Lees ${ }^{23}$ but valid only in the limit of zero Mach number. Using similar procedure but considering the real part of the resulting equation leads to the second and stricter necessary condition for instability: $(B)\left(\bar{U}_{z}-\bar{U}_{z c}\right) D \Omega\left(r_{c}\right)<0$, where $\bar{U}_{z c}$ is the velocity at $r=r_{c}$, where condition $(A)$ holds. This condition is again valid only in the limit of zero Mach number.
For neutral stability ( $\left.\omega_{i}=0\right)$, Eq. (9) has a singularity at $r=r_{s}$, where $\bar{U}_{z}=\omega_{r} / \alpha$, unless $D \Omega(r)=0$ at the point. This gives a necessary condition for the existence of neutral stability, independent of the Mach number.

It is seen that in general the stability of compressible flow is dependent on the Mach number. The existence of an inflection point as a necessary condition for instability cannot be obtained except for zero Mach number. An implication of this is that certain flow profiles without an inflection point, which do not show instability in incompressible flow, may be unstable in compressible flow. This applies to the profiles of Eqs. (7) and (8) for axisymmetric waves $(m=0)$, where there is no interior point at which $D \Omega(r)=0$. However, for helical waves $(m \neq 0), \Omega(0)$ $=\Omega(\infty)=0$, there must be an interior point where $D \Omega=0$, regardless of the exact form of the velocity and temperature profiles. The profiles of Eqs. (6) and (8) satisfy both conditions (A) and (B) for any azimuthal mode $m$.

\section{INSTABILITIES OF “TOP-HAT”' JETS}

The stability characteristics of the "top-hat" jet profile are presented in this section. Compressibility and threedimensionality effects are studied by varying the jet Mach number $M_{1}$ and the azimuthal mode number $m$. Except otherwise stated, the spatial stability problem is assumed. The jet parameter $R_{z}$ is 10 and the ambient fluid is at rest $\left(\bar{U}_{2}=0\right)$ with the same mean temperature as the jet fluid $\left(\bar{T}_{2}=1\right)$. (Without ambiguity, the jet Mach number will be written simply as $M$ whenever the ambient Mach number $M_{2}$ is zero in the following sections.) The Froude number is assumed to be infinity. The mean velocity profile is given by Eq. (6) and the temperature profile by Eq. (8).

As expected, both vortical and acoustic mode instabilities are found for this profile. Although vortical mode has been studied extensively in the past, it is presented here for comparison with acoustic modes. Figure 1 shows the spatial growth rate and phase speed of the vortical, first helical $(m=1)$ mode against frequency at various Mach numbers. It should be noted that for all helical modes, the plotted quantity $\omega / \alpha_{r}$ is the projection of the actual phase speed on the $z$-axis. As $M$ increases, both the maximum growth rate and the unstable frequency range decrease while the location of peak growth rate shifts to lower frequency. As frequency increases at a given $M$, the phase speed first decreases and then increases after reaching a minimum. These general characteristics are also true for the vortical, axisymmetric mode $(m=0)$ and higher helical modes $(m>1)$. Figure 2 shows the peak growth rate of different azimuthal modes against the Mach number. In all cases, the peak growth rate decreases rapidly with increasing $M$. However, the axisymmetric mode has the highest rate of decrease. As a result, the dominance of the axisymmetric mode at low Mach numbers gives way to helical modes at $M>1$. At large $M(>8)$, the first helical mode is the only remaining unstable mode. Thus for vortical mode, three-dimensional waves become more important as $M$ increases.

As indicated earlier, the wave phase speed relative to the free-streams is an important parameter as far as sound gen- 

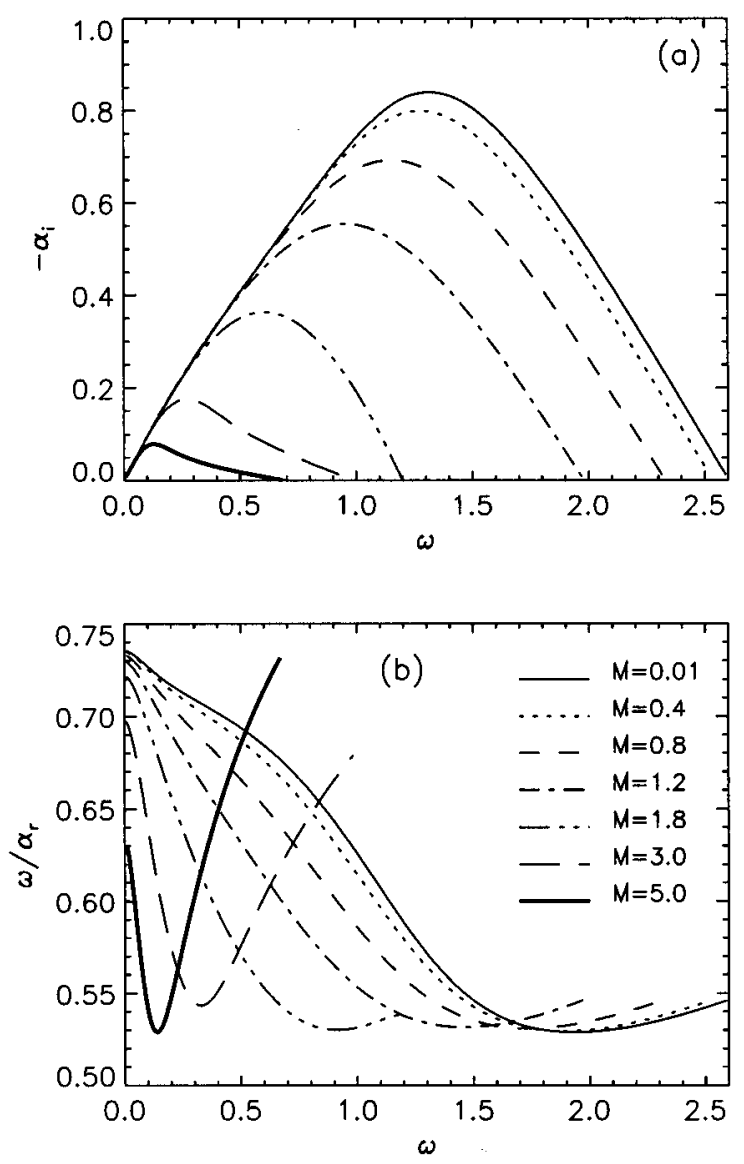

FIG. 1. The spatial growth rate (a) and the phase speed (b) of the vortical, first helical mode in a "top-hat" jet at different Mach numbers.

eration is concerned. In a quite different context, $\mathrm{Lin}^{24}$ introduced the concept of relative Mach numbers. In the present cylindrical coordinate system, the relative Mach numbers for the jet and the ambient streams are defined, respectively, as:

$$
M_{r 1}=M_{1} \cos \phi\left(\bar{U}_{1}-\frac{c_{p h}}{\cos \phi}\right),
$$

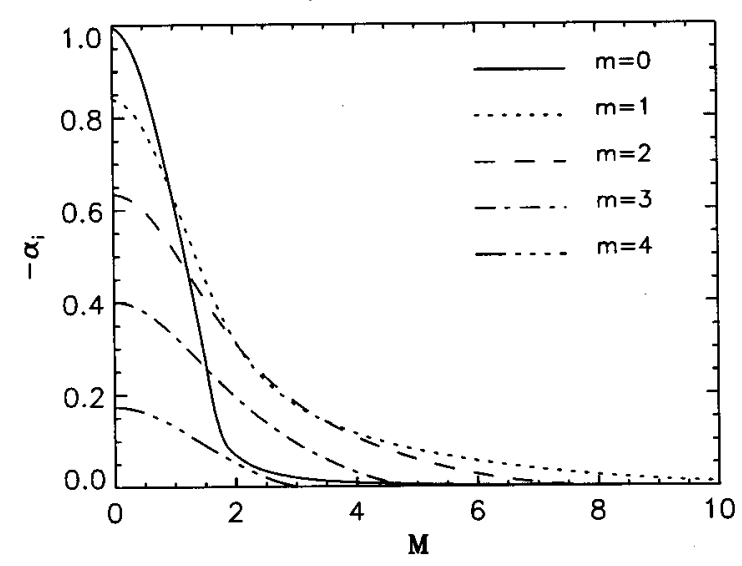

FIG. 2. Mach-number effects on the spatial growth rate of the vortical mode in a "top-hat" jet.
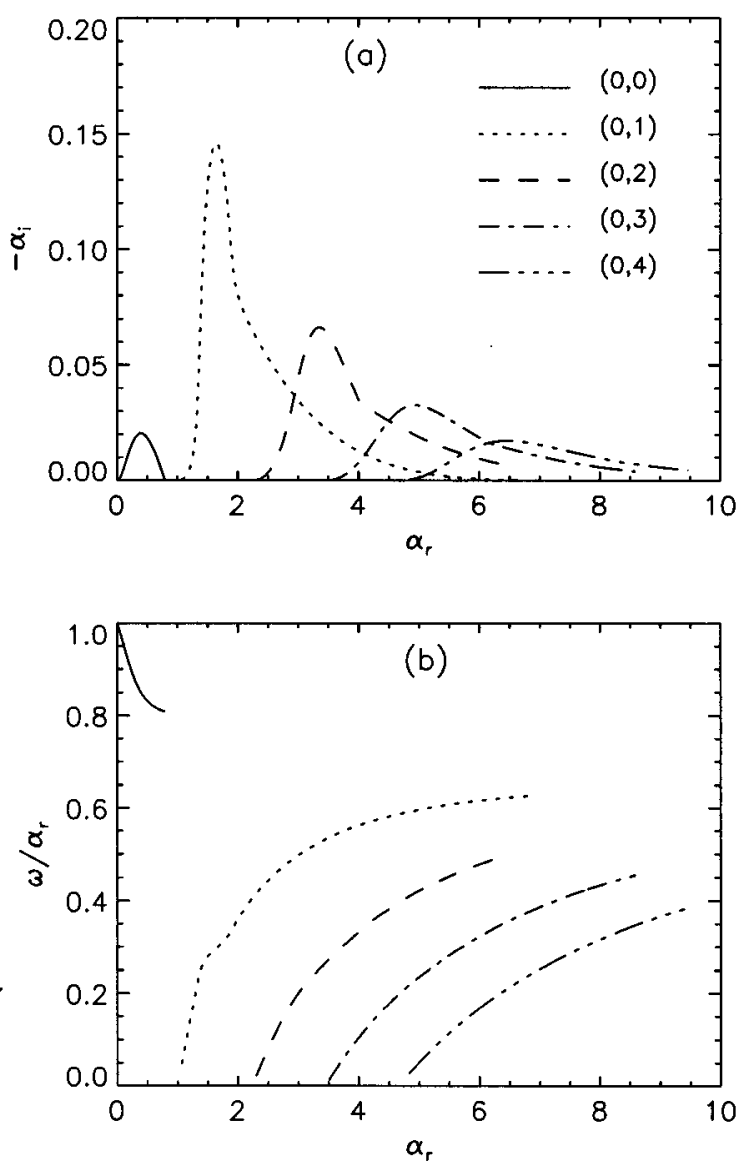

FIG. 3. The spatial growth rate (a) and the phase speed (b) of vortical and acoustic axisymmetric modes in a Mach 3 “top-hat" jet.

$$
M_{r 2}=\frac{M_{1}}{\bar{T}_{2}^{1 / 2}} \cos \phi\left(\frac{c_{p h}}{\cos \phi}-\bar{U}_{2}\right),
$$

where $\cos \phi=\alpha /\left(\alpha^{2}+m^{2} / r^{2}\right)^{1 / 2}$ based on Eq. (1). A relative Mach number is a wave Mach number in the direction of the wave traveling at an angle $\phi$ relative to the $z$-axis with phase speed $c_{\mathrm{ph}}$. A similar Mach number was defined by Mack ${ }^{17}$ in the Cartesian coordinate system. When $M_{r i}>1(i=1,2)$, the wave is said to be supersonic relative to stream $i$. When $M_{r i}<1(i=1,2)$, the wave is said to be subsonic relative to stream $i$. For axisymmetric waves in a jet with $\bar{U}_{2}=0$, $\bar{T}_{2}=1$, the relative Mach numbers reduce to $M_{r 1}$ $=M_{1}\left(1-c_{\mathrm{ph}}\right)$ and $M_{r 2}=M_{1} c_{\mathrm{ph}}$.

According to Mack, ${ }^{17}$ acoustic modes exist whenever there is an embedded region of locally supersonic flow relative to the phase speed of the instability wave. This is equivalent to the condition $M_{r 1}>1$ for jets. In Fig. 3, unstable modes in a Mach 3 jet $\left(\bar{U}_{2}=0\right)$ are shown. The growth rate and phase speed of axisymmetric waves are plotted against the real part of the axial wavenumber $\left(\alpha_{r}\right)$ rather than the frequency to better illustrate the different modes. For convenience of discussions, each continuous curve in the plot is designated by an integer pair $(m, n)$. Thus mode $(m, n)$ has an azimuthal wavenumber $m$ defined in Eq. (1) and an acoustic mode number $n$. Here, $n$ is an integer number starting from 0 and increasing by 1 for each curve in the 
$\alpha_{r}$-direction. Therefore, Fig. 3 contains modes from $(0,0)$ to $(0,4)$. The larger $n$ is, the higher the axial wavenumber (or frequency) at which the peak growth rate for that mode occurs. Since there could be an infinite spectrum of acoustic modes, ${ }^{4,17} n$ could be very large. However, the growth rate for modes with large $n$ is very small so that it is sufficient to plot only the first few modes.

As is clearly seen in Fig. 3, mode $(0,0)$ is very different from other modes. Its phase speed indicates that it is subsonic to the jet stream $\left(M_{r 1}<1\right)$ and supersonic to the ambient stream $\left(M_{r 2}>1\right)$. Hence, by Mack's criterion, it is not an acoustic mode. To trace the origin of mode $(0,0)$, the jet Mach number $M$ was systematically reduced and mode $(0,0)$ was seen merging with the Kelvin-Helmholtz mode at $M=0$. Hence, mode $(0,0)$ is a continuation of the KelvinHelmholtz instability into the supersonic regime and thus a vortical mode. On the other hand, modes $(0,1)$ to $(0,4)$ are supersonic to the jet stream $\left(M_{r 1}>1\right.$ for $\left.\omega / \alpha_{r}<2 / 3\right)$ and Mack's criterion for acoustic modes is satisfied. Furthermore, these modes do not continue down to the subsonic regime $(M<1)$ if $M$ is systematically reduced. They are thus acoustic modes. By examination of the phase speed, each of these modes has two regimes: one supersonic $\left(M_{r 2}>1\right.$ for $\left.\omega / \alpha_{r}>1 / 3\right)$ and the other subsonic $\left(M_{r 2}<1\right.$ for $\left.\omega / \alpha_{r}<1 / 3\right)$ to the ambient. In Fig. 4, four types of characteristic pressure eigenfunctions are shown. Figures 4(c) and $4(d)$ are eigenfunctions of the acoustic mode $(0,1)$ with subsonic $\left(M_{r 2}<1\right)$ and supersonic $\left(M_{r 2}>1\right)$ phase speed, respectively. The pressure disturbance of an acoustic subsonic wave is basically confined within the jet. This conforms to the physical picture of an acoustic wave that is trapped within the jet reflecting back and forth. In contrast, the pressure disturbance of an acoustic supersonic wave oscillates to the far field and appears to be radiating. Nevertheless, the part of the eigenfunction within the jet is very similar to that of an acoustic subsonic wave, indicating its common origin. On the other hand, eigenfunctions of vortical modes shown in Figs. 4(a) and 4(b) are quite different. Figure 4(b) is for mode $(0,0)$ at $M=3$, which has supersonic phase speed $\left(M_{r 2}>1\right)$. It is of radiating nature but does not have the dominant part inside the jet typical of acoustic modes. Figure 4(a) is for a vortical mode in a subsonic jet at $M=0.8$. The eigenfunction decays rapidly to zero just outside of the jet. Within the jet, the shape of eigenfunction is very different from that of an acoustic mode. In the direct numerical simulations of Luo and Sandham, ${ }^{25}$ it was shown that those modes with radiating eigenfunctions, either of vortical or acoustic nature, were far more efficient in sound generation than modes with non-radiating eigenfunctions. The essential difference still lies with that of supersonic and subsonic phase speed. However, it is less ambiguous to use terms "radiating mode" and "non-radiating mode" in the context of jets since "radiating" implies radiation away from the jet into the ambient. To use "supersonic mode" and "subsonic mode," one always has to clarify to which free-stream the terms are referring. Based on the above practical reasoning, it is useful to classify instability waves into four modes: (I) non-radiating vortical, (II) radiating vortical, (III) nonradiating acoustic and (IV) radiating acoustic.
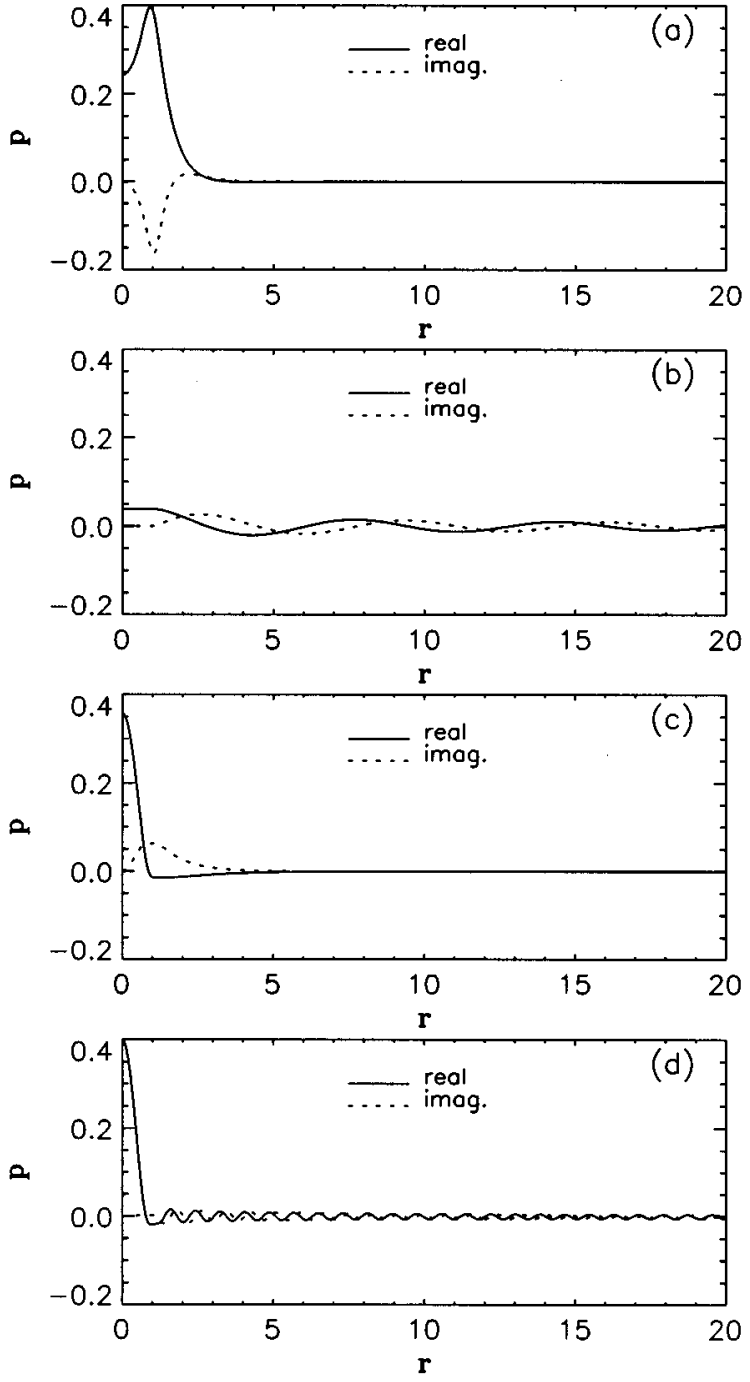

FIG. 4. Pressure eigenfunctions of (a) a non-radiating vortical wave at $M=0.8$; (b) a radiating vortical wave at $M=3.0$; (c) a non-radiating acoustic wave at $M=3.0$; (d) a radiating acoustic wave at $M=3.0$.

Acoustic modes are also found for helical modes $(m \neq 0)$ at Mach 3. Figure 5 shows first helical modes. The distribution of these modes in the wavenumber space is very similar to that of the axisymmetric modes but there are differences. For axisymmetric waves, the vortical mode is less unstable than the leading acoustic modes. For helical waves, the vortical mode is more unstable than any acoustic mode. Also, the relative Mach numbers defined in Eqs. (12) and (13) are functions of phase angle $\phi$ or $r$. It can be seen that the vortical mode can be supersonic $\left(M_{r 1}>1\right)$ or subsonic $\left(M_{r 1}<1\right)$ to the jet stream. Near the jet axis $(r \rightarrow 0), M_{r 1}$ becomes very small. The maximum growth rate and the corresponding wavenumber and frequency at $M=3$ for $m=0,1,2$ are shown in Tables I to III. The following characteristics are observed: (a) for the vortical mode, helical waves are more unstable than the axisymmetric wave but the opposite is true for acoustic modes; (b) the acoustic first helical mode is more unstable than acoustic higher helical $(m>1)$ modes; (c) acoustic modes generally have larger wavenumbers than the vortical mode; (d) peak growth rates 

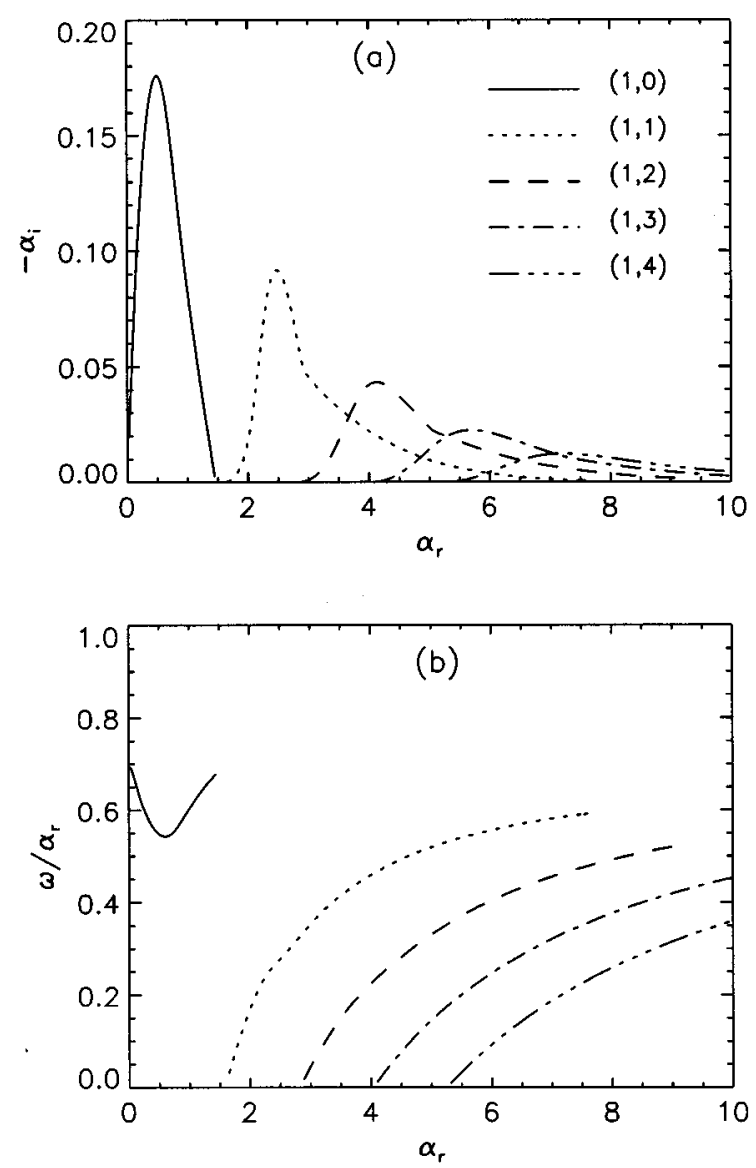

FIG. 5. The spatial growth rate (a) and the phase speed (b) of vortical and acoustic first helical modes in a Mach 3 "top-hat" jet.

of acoustic modes occur at almost equally spaced wavenumbers; (e) peak growth rates of acoustic modes occur in the subsonic regime $\left(M_{r 2}<1\right)$ with relatively constant phase speed.

The higher acoustic modes $(n>1)$ have the same physical origin as the first acoustic mode $(n=1)$ but shorter wavelengths. Therefore, a larger number of wavelengths can fit into the edges of the jet. ${ }^{10}$ As a result, there are correspondingly more nodes and antinodes or zero-crossings in their pressure eigenfunctions. Such phenomena have been shown by Tam and $\mathrm{Hu}^{4}$ and Mack ${ }^{17}$ and are not repeated here. Nevertheless, an example of the second acoustic mode is provided in Fig. 13(c) in the context of a fully developed jet to be discussed later. It is noted that the number of zero-

TABLE I. Maximum growth rates of vortical and acoustic instability modes for $m=0$ at Mach 3 .

\begin{tabular}{ccccc}
\hline \hline$(m, n)$ & $\omega$ & $\alpha_{r}$ & $-\alpha_{i}$ & $\omega / \alpha_{r}$ \\
\hline$(0,0)$ & 0.34 & 0.401 & 0.0204 & 0.848 \\
$(0,1)$ & 0.49 & 1.645 & 0.1459 & 0.298 \\
$(0,2)$ & 0.85 & 3.352 & 0.0662 & 0.254 \\
$(0,3)$ & 1.125 & 4.934 & 0.0326 & 0.228 \\
$(0,4)$ & 1.36 & 6.463 & 0.0173 & 0.210 \\
$(0,5)$ & 1.579 & 7.976 & 0.0095 & 0.198 \\
$(0,6)$ & 1.798 & 9.493 & 0.0053 & 0.189 \\
\hline \hline
\end{tabular}

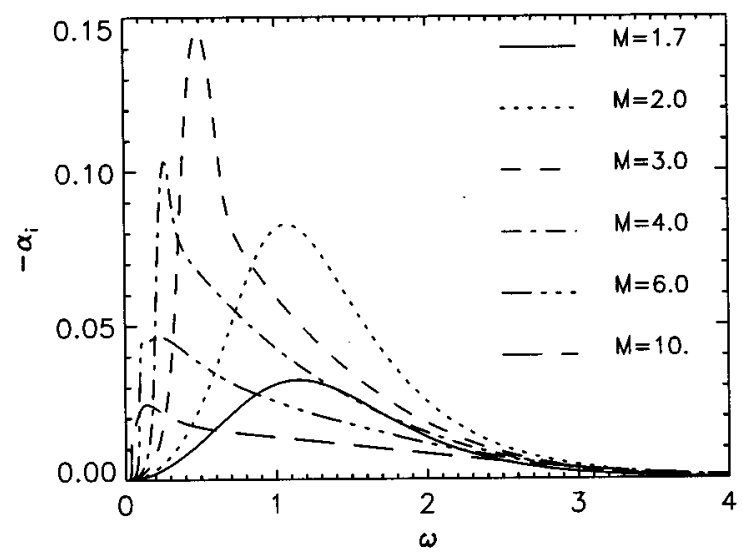

FIG. 6. The spatial growth rate of acoustic mode $(0,1)$ in a "top-hat' jet at different Mach numbers.

crossings in the pressure eigenfunction inside the jet corresponds uniquely to the mode number $n$. However, due to the existence of radiating acoustic modes, the total number of zero-crossings in the pressure eigenfunction is not unique.

The growth rate of the acoustic, axisymmetric mode $(0,1)$ at Mach numbers up to 10 is plotted in Fig. 6. The maximum growth rate of this mode occurs at $M=3$. When $M$ increases, the peak growth rate occurs at a lower frequency. Interestingly, the unstable frequency range changes little with $M$, contrary to the trend for vortical mode instability (Fig. 1). The peak growth rate at each Mach number for modes $(0,1),(0,2)$ and $(1,1)$ is plotted in Fig. 7. Acoustic modes are found for Mach numbers down to just over unity, but those with significant growth rates are in the range of $2<M<10$. The most unstable region is around $M=3$ for all three modes. Beyond $M=20$, the curves flatten out and appear to approach some constant value. Surprisingly, the peak growth rate of mode $(0,1)$ is less than that of mode $(0,2)$ for $5<M<17$ while Mack $^{17}$ found that the first acoustic mode was always the dominant one.

\section{TEMPERATURE-RATIO EFFECTS}

In the discussions of the previous section, the temperature of the jet (stream 1) and the ambient (stream 2) is kept equal, i.e., the temperature ratio $\bar{T}_{2} / \bar{T}_{1}=1$. However, as the Crocco-Busemann relation in Eq. (8) shows, aerodynamic heating has an influence on the mean temperature distribution and is dependent on the jet Mach number. Therefore,

TABLE II. Maximum growth rates of vortical and acoustic instability modes for $m=1$ at Mach 3 .

\begin{tabular}{ccccc}
\hline \hline$(m, n)$ & $\omega$ & $\alpha_{r}$ & $-\alpha_{i}$ & $\omega / \alpha_{r}$ \\
\hline$(1,0)$ & 0.27 & 0.491 & 0.1760 & 0.550 \\
$(1,1)$ & 0.69 & 2.488 & 0.0916 & 0.277 \\
$(1,2)$ & 1.00 & 4.138 & 0.0432 & 0.242 \\
$(1,3)$ & 1.25 & 5.694 & 0.0226 & 0.220 \\
$(1,4)$ & 1.47 & 7.210 & 0.0123 & 0.204 \\
$(1,5)$ & 1.69 & 8.728 & 0.0069 & 0.194 \\
$(1,6)$ & 1.91 & 10.249 & 0.0039 & 0.186 \\
\hline \hline
\end{tabular}




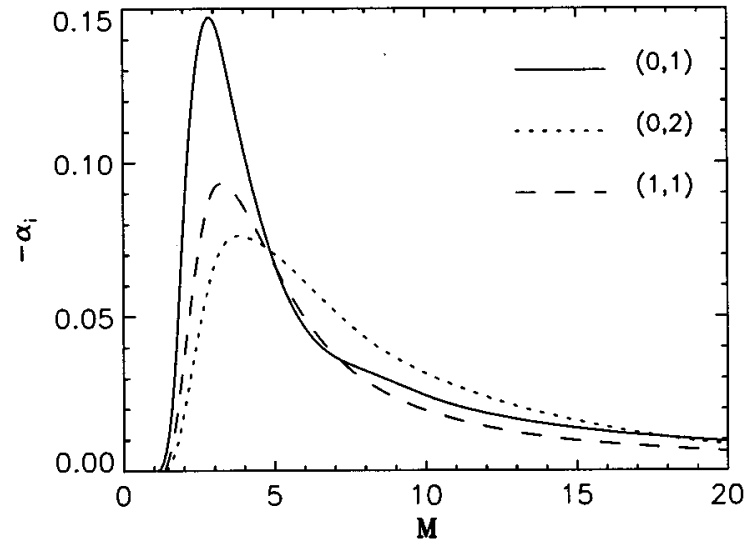

FIG. 7. Mach-number effects on the spatial growth rate of acoustic modes $(0,1),(0,2)$ and $(1,1)$ in a "top-hat" jet.

results of the previous section have already embodied the temperature effects. In this section, the temperature ratio (simply equal to $\bar{T}_{2}$ after normalization) is varied alone and other flow conditions are kept the same as described in the previous section. The objective is to clarify the effects of $\bar{T}_{2}$ on different jet instability modes.

Figure 8 shows the growth rate and phase speed of the vortical mode in a subsonic jet $M=0.8$ as $\bar{T}_{2}$ varies from 0.1 (hot jet) to 10 (cold jet). Compared with the $\bar{T}_{2}=1$ case, increasing $\bar{T}_{2}$ decreases the peak growth rate but broadens the unstable frequency range. On the other hand, decreasing $\bar{T}_{2}$ first increases and then decreases the peak growth rate. Decreasing $\bar{T}_{2}$ always narrows the unstable frequency range. In all cases, the phase speed over the whole frequency range is increased as $\bar{T}_{2}$ increases. Quite different trends are observed for vortical mode in a supersonic jet with $M=3$ as shown in Fig. 9. Compared with the $\bar{T}_{2}=1$ case, increasing $\bar{T}_{2}$ first increases and then decreases the peak growth rate but the unstable frequency range always broadens. Decreasing $\bar{T}_{2}$, on the other hand, always decreases the peak growth rate and the unstable frequency range. As to the phase speed, increasing $\bar{T}_{2}$ not only increases its overall magnitude but also alters its shape dramatically. It becomes clear that the temperature-ratio effects are coupled with compressibility.

The temperature-ratio effects on acoustic modes are shown in Fig. 10 for a Mach 3 jet. Surprisingly, the effects of varying $\bar{T}_{2}$ on the growth rate are qualitatively the same as on the vortical mode in the $M=0.8$ jet. However, the un-

TABLE III. Maximum growth rates of vortical and acoustic instability modes for $m=2$ at Mach 3 .

\begin{tabular}{ccccc}
\hline \hline$(m, n)$ & $\omega$ & $\alpha_{r}$ & $-\alpha_{i}$ & $\omega / \alpha_{r}$ \\
\hline$(2,0)$ & 0.317 & 0.660 & 0.1818 & 0.480 \\
$(2,1)$ & 0.86 & 3.246 & 0.0494 & 0.227 \\
$(2,2)$ & 1.13 & 4.871 & 0.0265 & 0.232 \\
$(2,3)$ & 1.37 & 6.429 & 0.0147 & 0.213 \\
$(2,4)$ & 1.59 & 7.955 & 0.0083 & 0.200 \\
$(2,5)$ & 1.81 & 9.481 & 0.0048 & 0.191 \\
$(2,6)$ & 2.03 & 11.007 & 0.0027 & 0.184 \\
\hline \hline
\end{tabular}
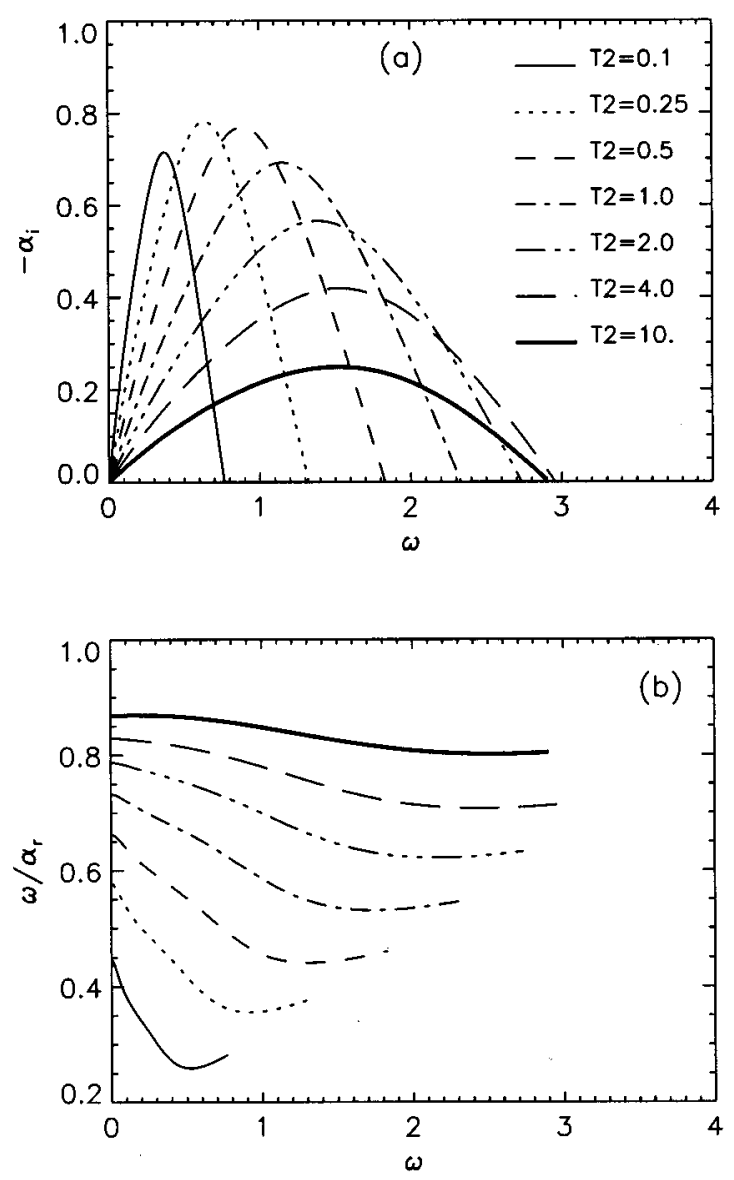

FIG. 8. Temperature-ratio effects on (a) the spatial growth rate and (b) the phase speed of the vortical mode $(1,0)$ in a Mach 0.8 "top-hat" jet.

stable frequency range is less sensitive to $\bar{T}_{2}$ in the present case, although the frequency range with substantial growth rate does narrow with decreasing $\bar{T}_{2}$. The most striking feature is that the phase speed of the acoustic mode is almost independent of $\bar{T}_{2}$, unlike the trend observed for any vortical mode. Also, the phase speed rises monotonically with frequency, which is a common character for all acoustic modes. According to Eq. (13), with constant phase speed, decreasing $\bar{T}_{2}$ will increase the relative Mach number $M_{r 2}$. Since higher $M_{r 2}$ will increase the tendency of jets to emit Mach waves, increasing the jet temperature (decreasing $\bar{T}_{2}$ ) would increase jet noise. This is in agreement of experimental observations by Oertel ${ }^{7,8}$ that hot jets produce more sound.

\section{INSTABILITIES OF FULLY DEVELOPED JETS}

The numerical procedures for solving the stability problem of the fully developed jet profile are the same as for the "top-hat" profile. The mean velocity profile is specified by Eq. (7), which has no adjustable parameter. The mean temperature is again calculated by the Crocco-Busemann relation in Eq. (8) with $\bar{T}_{2}=1$.

The investigation started with search for vortical instability of various azimuthal modes $m$. As expected, only helical waves were found to be unstable. Figure 11 shows the growth rate and phase speed of the vortical, first helical 

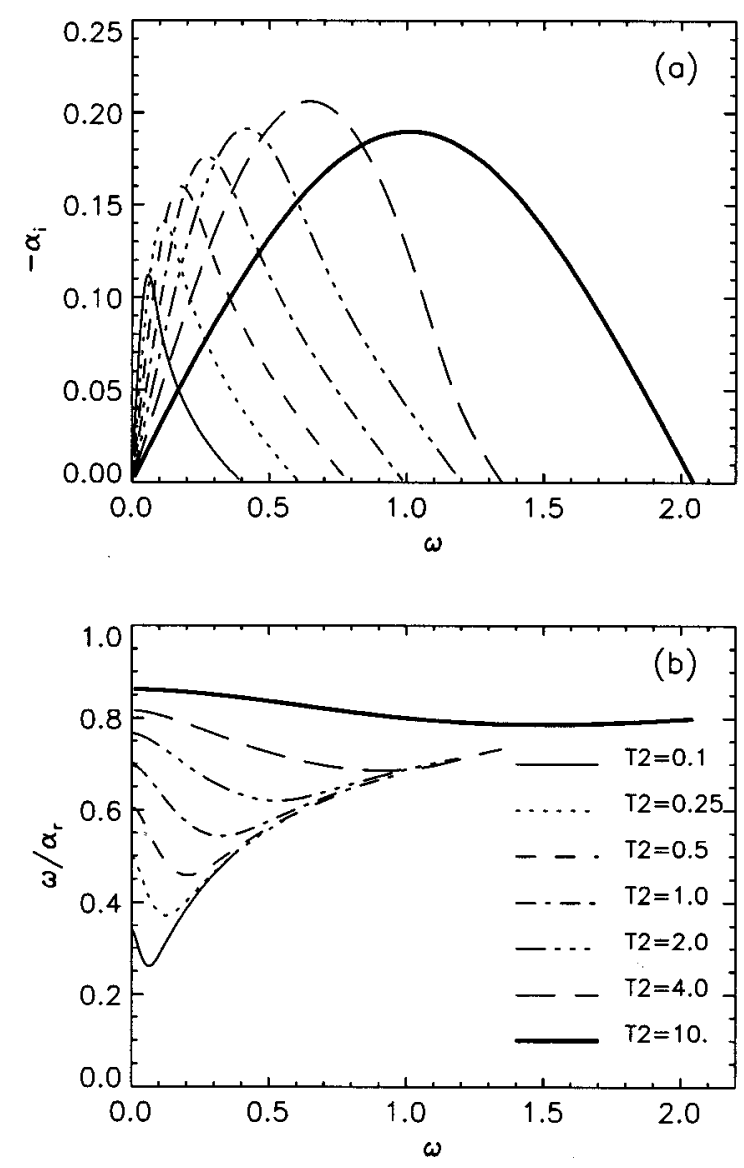

FIG. 9. Temperature-ratio effects on (a) the spatial growth rate and (b) the phase speed of the vortical mode $(1,0)$ in a Mach 3 "top-hat" jet.

mode $(1,0)$ at various Mach numbers. As for the "top-hat" jet, as $M$ increases, both the growth rate and unstable frequency range decrease while the location of peak growth rate shifts to lower frequency. However, the growth rate for the fully developed jet is much smaller at corresponding Mach numbers. The growth rate and frequency range of instability at $M=2$ are already very small and will further decrease as $M$ increases. The phase speed generally decreases with increasing $M$. However, it increases monotonically with frequency, which is quite different from the trend observed for the "top-hat" jet.

As discussed earlier, no amplified axisymmetric waves exist in incompressible jets with the fully developed profile. The situation is quite different in the present compressible jet. Figure 12 shows the growth rate and phase speed of unstable axisymmetric waves in the fully developed jet at three Mach numbers. With the same identifying procedures as described in Sec. IV, these unstable waves are found to belong to acoustic mode $(0,1)$. Unlike the same mode in the "top-hat" jet, the present one becomes more unstable as $M$ increases from 3 to 10 , although further increase in $M$ will result in a slight decrease in the growth rate. A more striking feature is that while the growth rate at $M=3$ has a single peak, more and more local peaks appear with increasing $M$. Notice that a small kink exists at about $\omega=1.2$ in the $M=3$ growth rate and reappear at $\omega=1.9$ in the $M=4$ growth rate. Such a kink usually marks the beginning of
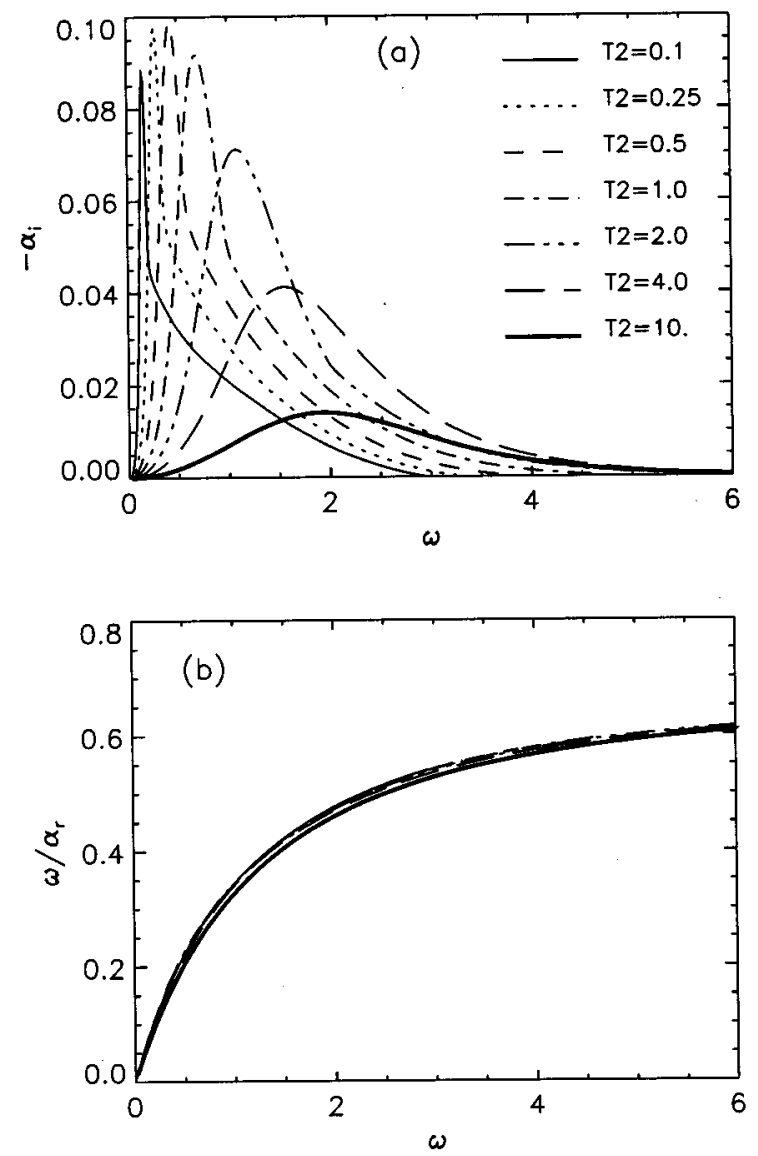

FIG. 10. Temperature-ratio effects on (a) the spatial growth rate and (b) the phase speed of the acoustic mode $(1,1)$ in a Mach 3 "top-hat" jet.

"mode mixing" as described by Michalke. ${ }^{13}$ Here, "mode mixing" refers to a merging of irregular modes with regular or normal unstable modes without passing through a neutral point. No satisfactory physical explanation is available for "mode mixing." The fact that local peaks and valleys in the growth rate occur at equally-spaced frequencies (e.g., at $M=10$ ), suggests a link with acoustic resonance. In the meantime, phase speeds at all Mach numbers are smooth, increasing functions of frequency, as shown in Fig. 12(b). It is noted that the phase speed of acoustic modes over the whole frequency range increases with $M$, in opposite trend to that of the vortical mode. The same phenomena are also observed in higher acoustic modes and in helical modes.

It is worth pointing out that oscillations in the growth rate curves are not caused by point-to-point numerical errors. Each curve presented here contains thousands of calculation points. At places of the kinks, calculations with different numerical procedures were conducted to ensure repeatability of the same phenomena.

The maximum growth rate of the vortical first helical mode $(1,0)$ at $M=2.0$ is $-\alpha_{i}=4.776 \times 10^{-4}$ (see Fig. 11) and even smaller at $M=3.0$. On the other hand, the peak growth rate of the acoustic axisymmetric mode $(0,1)$ at $M=3.0$ is $-\alpha_{i}=2.423 \times 10^{-3}$ (Fig. 12). This shows that amplified axisymmetric modes not only exist in the fully developed profile but also become dominant at high Mach numbers. 

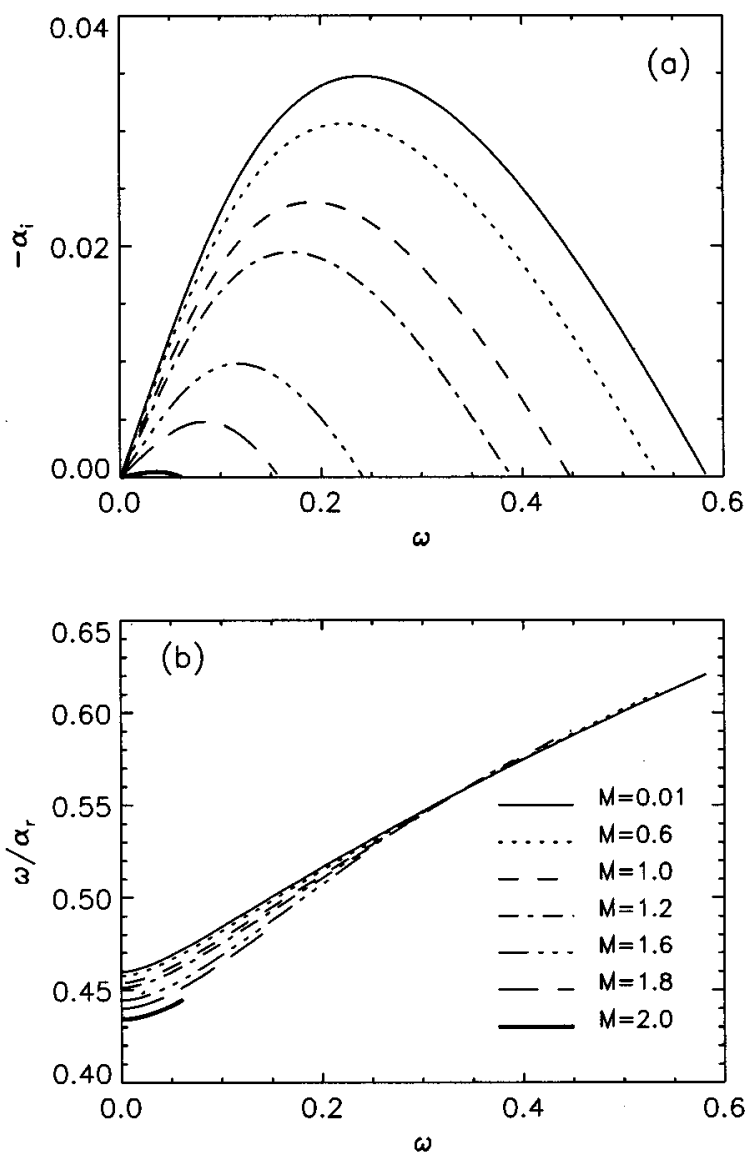

FIG. 11. The spatial growth rate (a) and the phase speed (b) of the vortical mode $(1,0)$ in a fully developed jet at different Mach numbers.

As in the case of the "top-hat" jet, there is a spectrum of unstable acoustic modes for the fully developed profile. Figure 13 shows only the growth rate of the acoustic axisymmetric mode $(0,2)$. The general trend with increasing $M$ is the same as for mode $(0,1)$ but the growth rate at a corresponding Mach number is generally lower. Calculations have also found spectra of unstable waves in helical modes $m \geqslant 1$ and higher acoustic modes $n \geqslant 1$ at different Mach numbers. The general characteristics are very similar to those of mode $(0,1)$ and thus not presented. Finally, some typical pressure eigenfunctions of acoustic modes in the fully developed jet are shown in Fig. 14. Again, non-radiating and radiating modes exist for each acoustic mode and those for mode $(0,1)$ are illustrated in Figs. 14(a) and 14(b). These are quite similar to corresponding eigenfunctions of the tophat' jet. Figure 14(c) shows the pressure eigenfunction of a non-radiating higher acoustic mode $(0,2)$. As explained before, one more zero-crossing exists in the eigenfunction of mode $(0,2)$ as compared with that of mode $(0,1)$. Nevertheless, the main part of the pressure disturbance is similar, indicating their common origin.

\section{DISCUSSION}

Vortical and acoustic mode instabilities have been found in both a "top-hat" and a fully developed jet profiles. Numerical results show that vortical and acoustic modes have
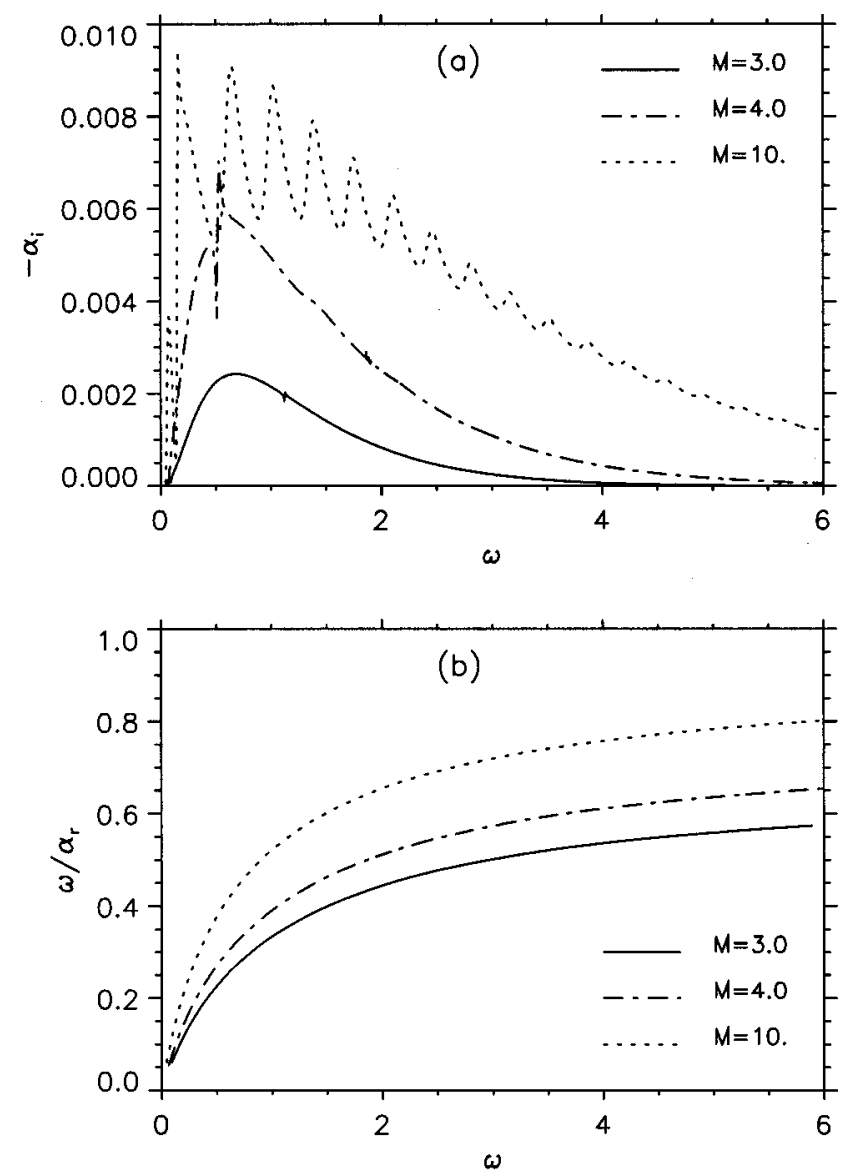

FIG. 12. The spatial growth rate (a) and the phase speed (b) of the axisymmetric, first acoustic mode $(0,1)$ in a fully developed jet at three Mach numbers.

quite different characteristics in terms of their existence conditions, their eigenfunction shapes, their responses to the Mach number, temperature ratio and velocity profile changes. However, a clear physical explanation of the mechanisms behind these modes does not exist, at least for jets with realistic thickness. For jets with thin shear layers which can be treated as a vortex sheet, several plausible arguments do exist such as the wavy-wall theory of Tam and

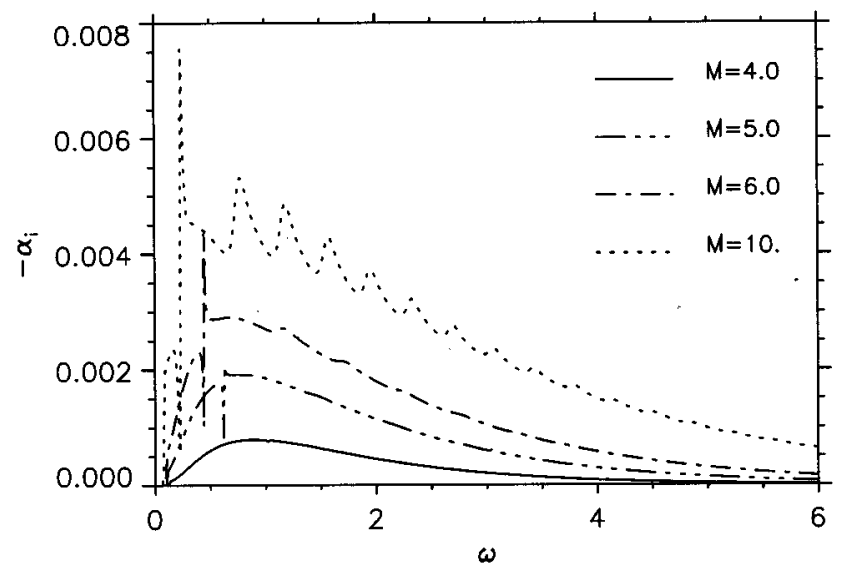

FIG. 13. The spatial growth rate of the axisymmetric, second acoustic mode $(0,2)$ in a fully developed jet at four Mach numbers. 

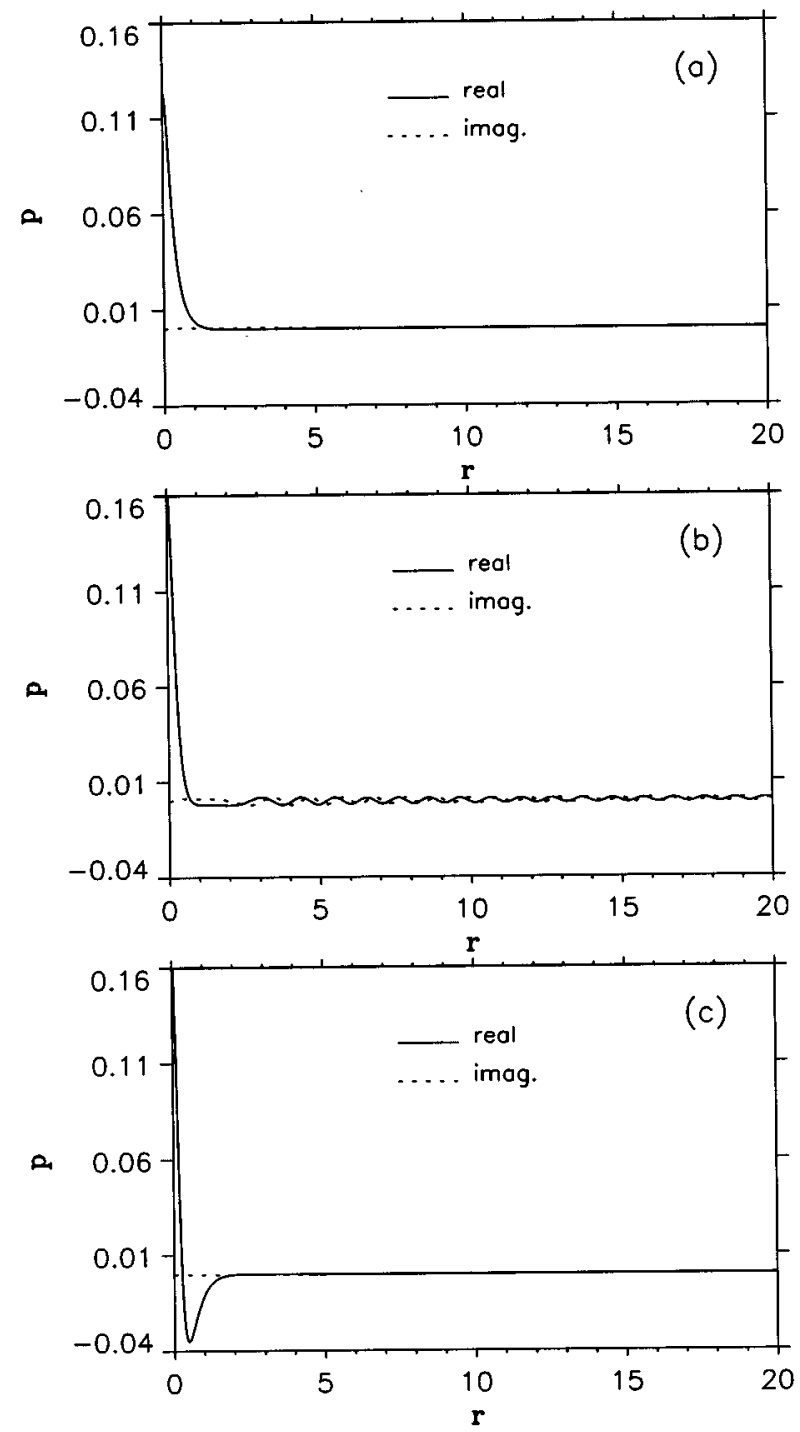

FIG. 14. Pressure eigenfunctions of unstable axisymmetric modes in a fully developed jet at $M=10$. (a) A non-radiating wave of the first acoustic mode $(0,1)$; (b) a radiating wave of the first acoustic mode $(0,1)$; (c) a nonradiating wave of the second acoustic mode $(0,2)$.

$\mathrm{Hu},{ }^{4}$ the wave reflection theory of Mack ${ }^{17}$ and the resonance theory of Miles ${ }^{26}$ and Gill. ${ }^{10}$ Tam and Hu's theory is based on Ackeret's explanation of the Kelvin-Helmholtz instability but they extend it to supersonic flow. It is assumed that a vortex sheet separating the jet flow and the ambient fluid is initially deformed by a Kelvin-Helmholtz wave with a phase speed $c_{\mathrm{ph}}$. The vortex sheet is then regarded as a wavy wall in a reference frame traveling with speed $c_{\mathrm{ph}}$. Analytical solutions of the pressure distributions on both sides of the wavy wall can be obtained. It is shown that in a subsonic jet, the pressure imbalance across the jet is in phase with the vortex-sheet displacement and the initial displacement would be augmented leading to the Kelvin-Helmholtz instability. In a supersonic jet where $c_{\mathrm{ph}}$ is still subsonic to the ambient fluid, the pressure imbalance is $180^{\circ}$ out of phase with the displacement and only neutrally stable waves may exist. (But they point out that in a finite-thickness jet, unstable "acoustic" subsonic waves can exist.) When the jet Mach number is high enough so that $c_{\mathrm{ph}}$ is supersonic to the ambient fluid, for certain wavelengths a pressure imbalance in phase with the vortex-sheet displacement is possible and unstable "acoustic" supersonic waves can exist. The attraction of the theory is that it offers a unified description of the vortical and acoustic instabilities. A drawback is that it excludes coexistence of the three modes at the same Mach number, which is disproved by our numerical results. Mack's physical picture of an acoustic mode is a sound wave trapped in a jet reflecting back and forth inside the jet boundaries. This offers a mechanism for a neutral wave but not an unstable wave. The theory of Miles and Gill is very similar to that of Mack but they speculate that sound waves impinging on the vortex sheet at certain angles cause a resonance that releases large amount of energy to support the back and forth movement of the neutral waves.

The above theories have provided some useful physical insight into the vortical and acoustic modes but none of them can fully explain the numerical results in previous sections. In an earlier paper by Kovásznay, ${ }^{27}$ three modes of disturbance fields are identified in compressible viscous flow: vorticity mode, sound-wave mode and entropy mode. The first two modes of disturbances would lead to the vortical and acoustic instability modes described in this study. The entropy mode, which is caused by density changes due to heat conduction, is missing from the present inviscid analysis. According to Kovásznay, ${ }^{27}$ the three modes are independent when the fluctuations are weak but they interact under stronger fluctuations. In the present linear context, the vortical and acoustic modes are expected to act independently and thus can co-exist at the same Mach number. Since vorticity disturbances are present regardless of the Mach number, it is not surprising that vortical instability exists in incompressible and compressible flow. On the other hand, sound-wave disturbances are only a compressible flow phenomenon so that acoustic instability exists in compressible flow only. Furthermore, when acoustic waves impinge on a vortex sheet or a wavy wall as in Tam and Hu's theory, they act directly as "imposed" pressure distributions along the sheet. Clearly, in a time-space transformation, these pressure distributions only depend on the frequency of the impinging waves at a given Mach number. At certain frequencies, the pressure distributions may cause a pressure imbalance across the vortex sheet that is in phase with the vortex-sheet displacement. This is when resonance occurs and instability arises. For a vortex sheet or a thin shear layer, a peak growth rate corresponds to a frequency at which the pressure imbalance is perfectly in phase with the vortex-sheet displacement. When there is a partial in-phase at other frequencies, a lower growth rate is obtained. In the above physical picture, the existence of an inflection point is irrelevant since acoustic waves act directly as a pressure imbalance that only depends on the wave frequency. And since complete out-of-phase between the pressure imbalance and the vortex sheet is so rare, it is not surprising that acoustic modes are unstable over a much larger frequency range compared with the vortical mode.

The above discussions can be extended to the peak-andvalley pattern of the growth rate curves of the fully devel- 
oped jet. As explained above, for a thin shear layer or a vortex sheet, one peak growth rate is expected when the pressure imbalance across the layer is perfectly in phase with the layer displacement at a resonance frequency. A thick shear layer such as the fully developed jet profile may be viewed as an ensemble of such thin shear layers with cascading properties, each having an intrinsic resonance frequency and each giving a local peak growth rate. The result is an ensemble of local peaks with diminishing magnitudes at almost equally-spaced frequencies. As the Mach number is increased, the acoustic wavelength becomes smaller compared with the layer thickness so that progressively more peaks and valleys appear in the growth rate curves. As examples, the frequency differences $(\Delta \omega)$ between the local peaks or valleys (excluding the first two at low frequencies) are calculated, which show extraordinary regularity for a given mode and a Mach number. For mode $(0,1)$ at $M=10, \Delta \omega=0.37$. For mode $(0,2)$ at $M=10, \Delta \omega=0.40$. For mode $(0,1)$ at $M=25, \Delta \omega=0.30$. The data actually show that as $M$ increases from 10 to $25, \Delta \omega$ decreases, which should be expected following the above discussions.

\section{CONCLUSIONS}

A linear stability theory for compressible flow has been formulated to study the stability properties of a "top-hat" and a fully developed jet profile at various Mach numbers and temperature ratios. Two types of instabilities have been found: vortical and acoustic. They have different existence conditions, different eigenfunction shapes and different responses to changes in the Mach number, temperature ratio and velocity profile. These differences are attributed to the different mechanisms behind the two instabilities. The vortical mode is due to the Kelvin-Helmholtz instability, which exists in compressible as well as incompressible flow. The acoustic mode is due to the direct action of acoustic waves on the shear layer through resonance, which exists only in compressible flow.

Numerical results in the "top-hat" jet have revealed a spectrum of acoustic modes for Mach numbers over unity. Both acoustic and vortical modes can be radiating or nonradiating, depending on a relative Mach number. While the vortical mode dominates at low Mach numbers, it is the acoustic modes that dominate at high Mach numbers. In between the two modes co-exist and have growth rates of the same order of magnitude. It has been observed that the second acoustic mode can be more unstable than the first acoustic mode in some Mach number range. Temperature-ratio effects, which can be stabilizing or destabilizing, are strongly linked with the Mach number and mode of instability.

Unstable vortical and acoustic modes in the fully developed profile have been presented. In agreement with previous studies, no axisymmetric vortical waves are unstable. However, unstable axisymmetric acoustic waves do exist and become dominant over both vortical and acoustic helical waves at Mach numbers over about 3. Strong evidence of a resonance mechanism for acoustic modes is seen in the growth rate curves at high Mach numbers, where a spectrum of local peaks and valleys appear at regularly-distributed frequencies.

\section{ACKNOWLEDGMENTS}

The work was funded by the U.K. Engineering and Physical Sciences Research Council under Grant No. GR/ H40518.

${ }^{1}$ C. K. W. Tam, "Directional acoustic radiation from a supersonic jet generated by shear layer instability," J. Fluid Mech. 46, 757 (1971).

${ }^{2}$ T. R. Troutt and D. K. McLaughlin, "Experiments on the flow and acoustic properties of a moderate Reynolds number supersonic jets,' J. Fluid Mech. 116, 123 (1982).

${ }^{3}$ C. K. W. Tam and D. E. Burton, "Sound generated by instability waves of supersonic jets: Part 2, Axisymmetric jets," J. Fluid Mech. 138, 273 (1984).

${ }^{4}$ C. K. W. Tam and F. Q. Hu, "On the three families of instability waves of high-speed jets," J. Fluid Mech. 201, 447 (1989).

${ }^{5} \mathrm{C}$. K. W. Tam and P. Chen, "Relation between instability waves and noise of high-speed jets," AIAA J. 30, No. 7, 1747 (1992).

${ }^{6}$ C. K. W. Tam, "Supersonic jet noise,"' Annu. Rev. Fluid Mech. 27, 17 (1995).

${ }^{7}$ H. Oertel, "Mach wave radiation of hot supersonic jets," in Mechanics of Sound Generation in Flows, edited by E. A. Muller (Springer, New York, 1979), pp. 275-281.

${ }^{8} \mathrm{H}$. Oertel, "Mach wave radiation of hot supersonic jets investigated by means of the shock tube and new optical techniques," in Proceedings of the 12th International Symposium on Shock Tubes and Waves, Jerusalem, edited by A. Lifshitz and J. Rom (1980), pp. 266-275.

${ }^{9} \mathrm{H}$. Oertel, "Coherent structures producing Mach wave inside and outside of the supersonic jet," in Structure of Complex Turbulent Shear Flow, IUTAM Symposium, Marseille (1982).

${ }^{10}$ A. E. Gill, "Instabilities of 'top-hat' jets and wakes in compressible fluids," Phys. Fluids 8, 1428 (1965).

${ }^{11}$ L. Zaninetti, "Numerical results on instabilities of 'top-hat' jets," Phys. Fluids 29, 332 (1986).

${ }^{12}$ L. Zaninetti, "Maximum instabilities of compressible jets," Phys. Fluids 30, 612 (1987).

${ }^{13}$ A. Michalke, "Survey on jet instability theory," Prog. Aerospace Sci. 21, 159 (1984).

${ }^{14}$ G. K. Batchelor and E. A. Gill, "Analysis of the stability of axisymmetric jets," J. Fluid Mech. 14, 529 (1962).

${ }^{15} \mathrm{M}$. Lessen and P. J. Singh, "The stability of axisymmetric free shear layers," J. Fluid Mech. 60, 433 (1973).

${ }^{16} \mathrm{P}$. J. Morris, "The spatial viscous instability of axisymmetric jets," J. Fluid Mech. 77, 511 (1976)

${ }^{17}$ L. M. Mack, "On the inviscid acoustic-mode instability of supersonic shear flows. Part I: Two-dimensional waves," Theor. Comput. Fluid Dyn. 2, 97 (1990).

${ }^{18}$ L. M. Mack, "The stability of the compressible laminar boundary layer according to a direct numerical solution. Part IV," in Space Programs Summary, JPL, Pasadena, CA, 37, 271 (1963).

${ }^{19}$ L. D. Landau and E. M. Lifshitz, Fluid Mechanics (Pergamon, New York, 1959).

${ }^{20}$ Lord Rayleigh, "On the question of the stability of the flow of fluids," Scientific Papers (Cambridge University Press, Cambridge, 1892), Vol. 3, pp. $575-584$.

${ }^{21} \mathrm{R}$. Fjørtoft, "Application of integral theorems in deriving criteria of instability of laminar flow and for baroclinic circular vortex," Geofys. Publ. 17, No. 6 (1950).

${ }^{22}$ L. Lees and C. C. Lin, "Investigation of the stability of the laminar boundary layer in a compressible fluid," NACA Technical Note, No. 1115 (1946).

${ }^{23} \mathrm{~L}$. Lees, "The stability of the laminar boundary layer in a compressible fluid," NACA Technical Report, No. 876 (1947).

${ }^{24}$ C. C. Lin, "On the stability of the laminar mixing region between two parallel streams in a gas," NACA Technical Note, No. 2887 (1953).

${ }^{25}$ K. H. Luo and N. D. Sandham, "Acoustic wave and shear layer structures in a Mach 3 round jet," in Proceedings of the Tenth Symposium on Turbulent Shear Flow, Pennsylvania, August 14-16 (1995).

${ }^{26} \mathrm{~J}$. W. Miles, "On the reflection of sound at an interface of relative motion,'” J. Acoust. Soc. Am. 29, 226 (1957).

${ }^{27}$ L. S. G. Kovásznay, “Turbulence in supersonic flow,' J. Aeronaut. Sci. 20, 657 (1953). 